Supporting Information

\title{
A Wireless Implantable Strain Sensing Scheme using Ultrasound Imaging of Highly Stretchable Zinc Oxide/Poly Dimethylacrylamide Nanocomposite Hydrogel
}

Hongjie Jiang ${ }^{a b c}$, Natalie M. Carter ${ }^{d}$, Amin Zareei ${ }^{a d}$, Sina Nejati ${ }^{a d}$, Jose F. Waimin ${ }^{a d}$, Shirisha Chittiboyina $^{e}$, Elizabeth E. Niedert ${ }^{g}$, Tahereh Soleimani ${ }^{f}$, Sophie A. Lelièvre ${ }^{e}$, Craig J. Goergen ${ }^{g h}$, and Rahim Rahimi $a b d *$

${ }^{a}$ Birck Nanotechnology Center, Purdue University, West Lafayette, IN 47907-2057, USA

b School of Electrical and Computer Engineering, Purdue University, West Lafayette, IN 479072035, USA

c Shenzhen MSU-BIT University, Shenzhen, Guangdong, 518172, China

d School of Materials Engineering, Purdue University, West Lafayette, IN 47907-2045, USA

e Department of Basic Medical Sciences, Purdue University, 625 Harrison Street, West Lafayette, IN 47907, USA

${ }^{\mathrm{f}}$ College of Human Medicine, Michigan State University, East Lansing, MI, USA

g Weldon School of Biomedical Engineering, Purdue University, West Lafayette, IN 479072032, USA

${ }^{\text {h }}$ Center for Cancer Research, Purdue University, West Lafayette, IN 47907-2032, USA

*rrahimi@purdue.edu 

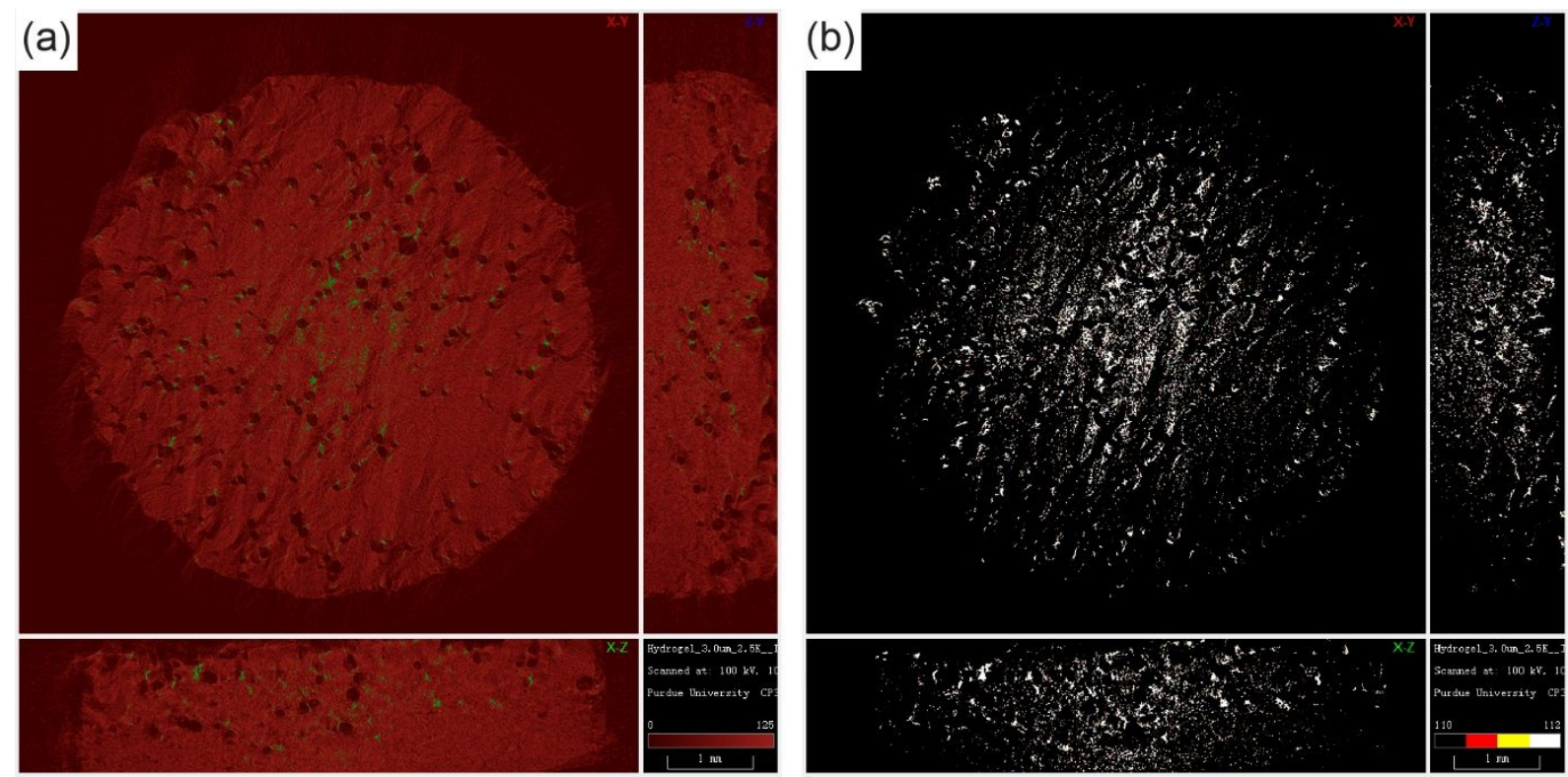

Figure S1. MicroCT scanning $10 \%$ gel with the cross-section at X-Y plane and the side-view at $\mathrm{X}-\mathrm{Z}$ and $\mathrm{Y}-\mathrm{Z}$ planes, showing the binary image (a) in color where the red indicated the gel matrix and the green represented the $\mathrm{ZnO}$ nanoparticles and (b) in black-white where the black indicated the gel matrix and the white represented the $\mathrm{ZnO}$ nanoparticles. 
(a) ultrasound visualization of $10 \% \mathrm{ZnO}-g e l$ under a frequency sweep of 20 to $50 \mathrm{MHz}$ in DI water, Agar, and cornAgar
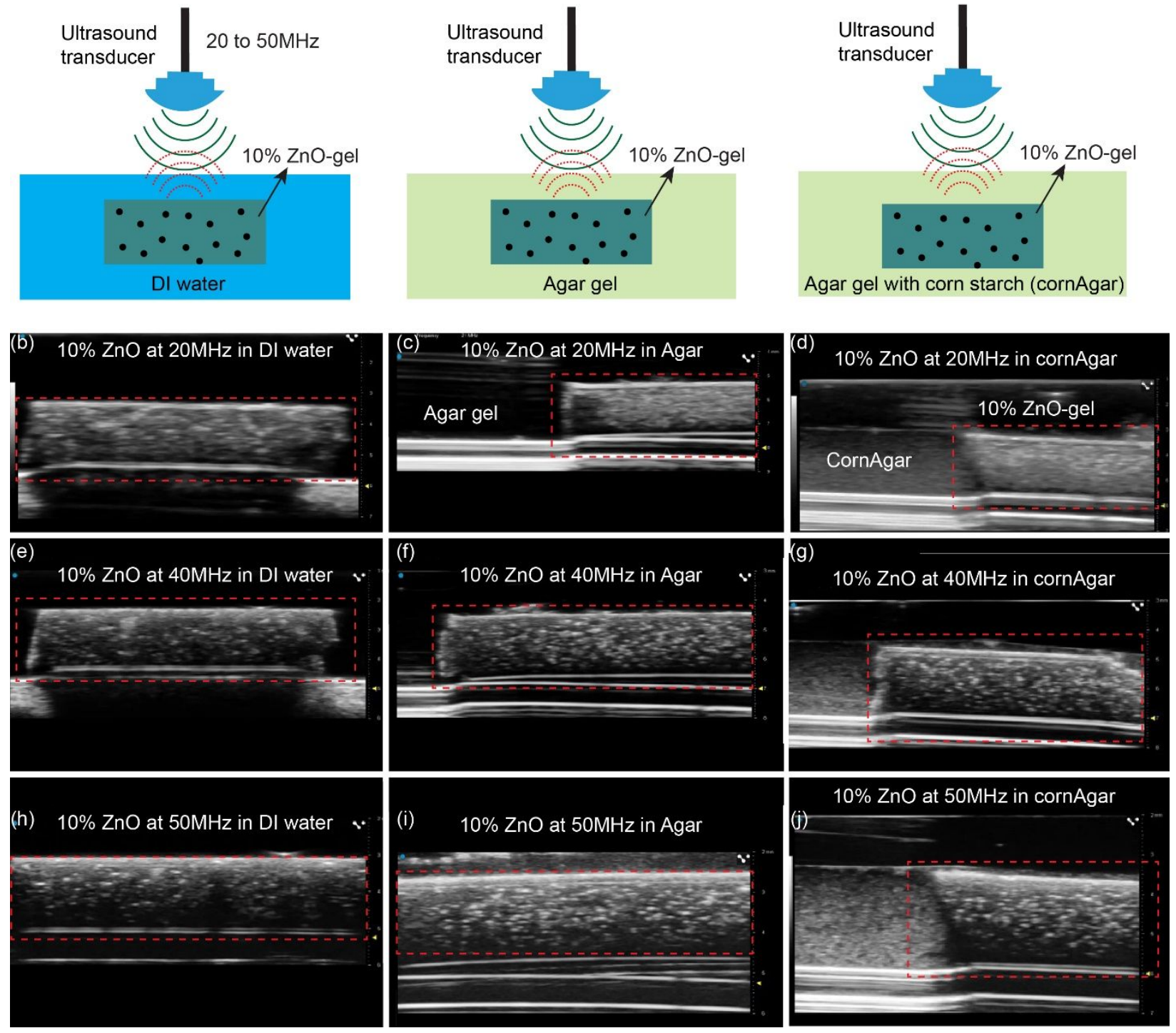

Figure S2. (a) Schematic illustration of ultrasound visualization of $10 \% \mathrm{ZnO}$-gel immersed in DI water, $0.5 \%$ Agar gel without and with $80 \%$ corn starch under an ultrasound frequency sweep of (b-d) 20MHz, (e-g) 40Mhz, and (h-j) 50Mhz. 
(a) ultrasound visualization of $40 \% \mathrm{ZnO}-g e l$ under a frequency sweep of 20 to $50 \mathrm{MHz}$ in DI water, Agar, and cornAgar
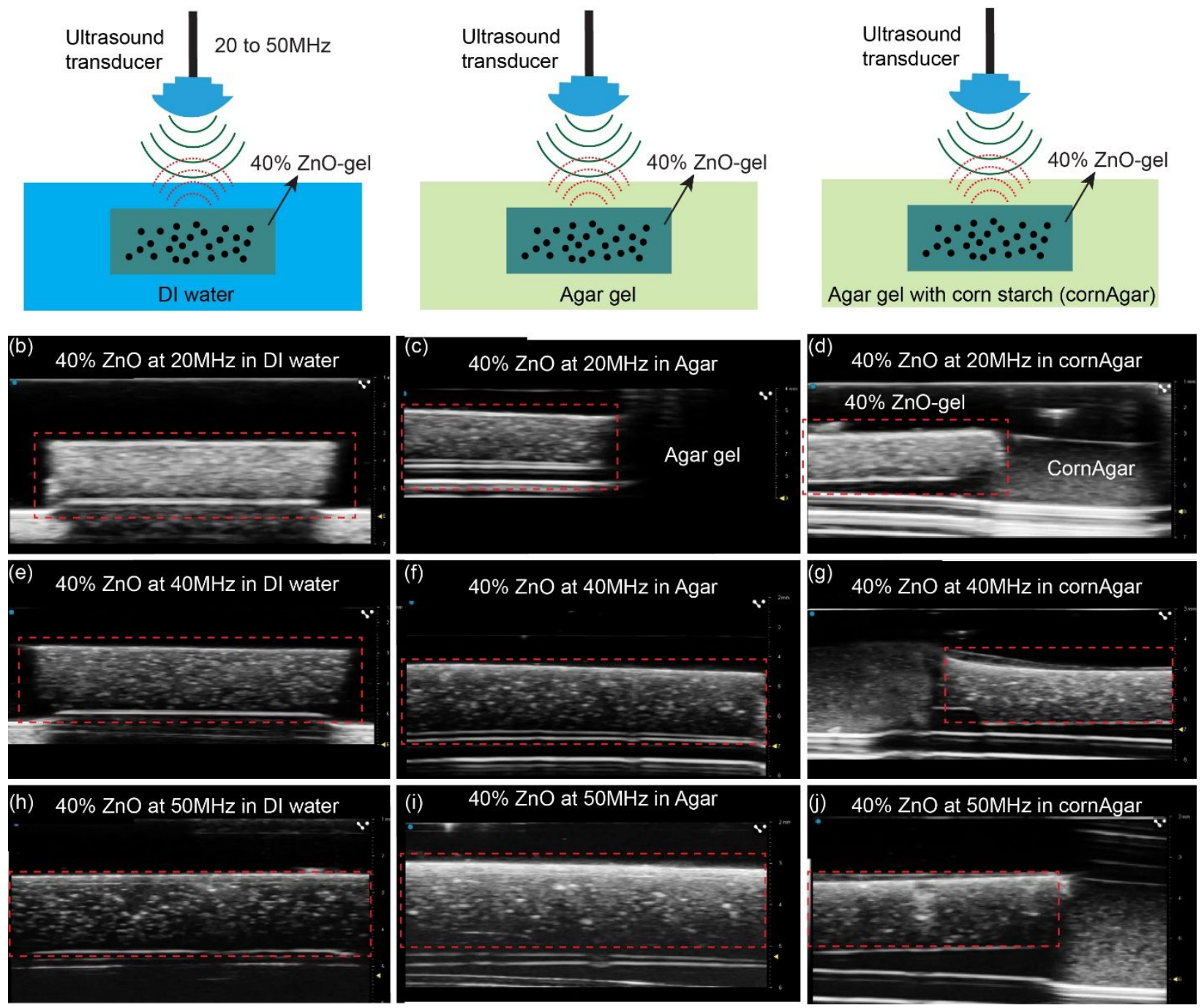

Figure S3. (a) Schematic illustration of ultrasound visualization of $40 \% \mathrm{ZnO}$-gel immersed in DI water, $0.5 \%$ Agar gel without and with $80 \%$ corn starch under an ultrasound frequency sweep of (b-d) $20 \mathrm{MHz},(\mathrm{e}-\mathrm{g}) 40 \mathrm{Mhz}$, and (h-j) 50Mhz. 


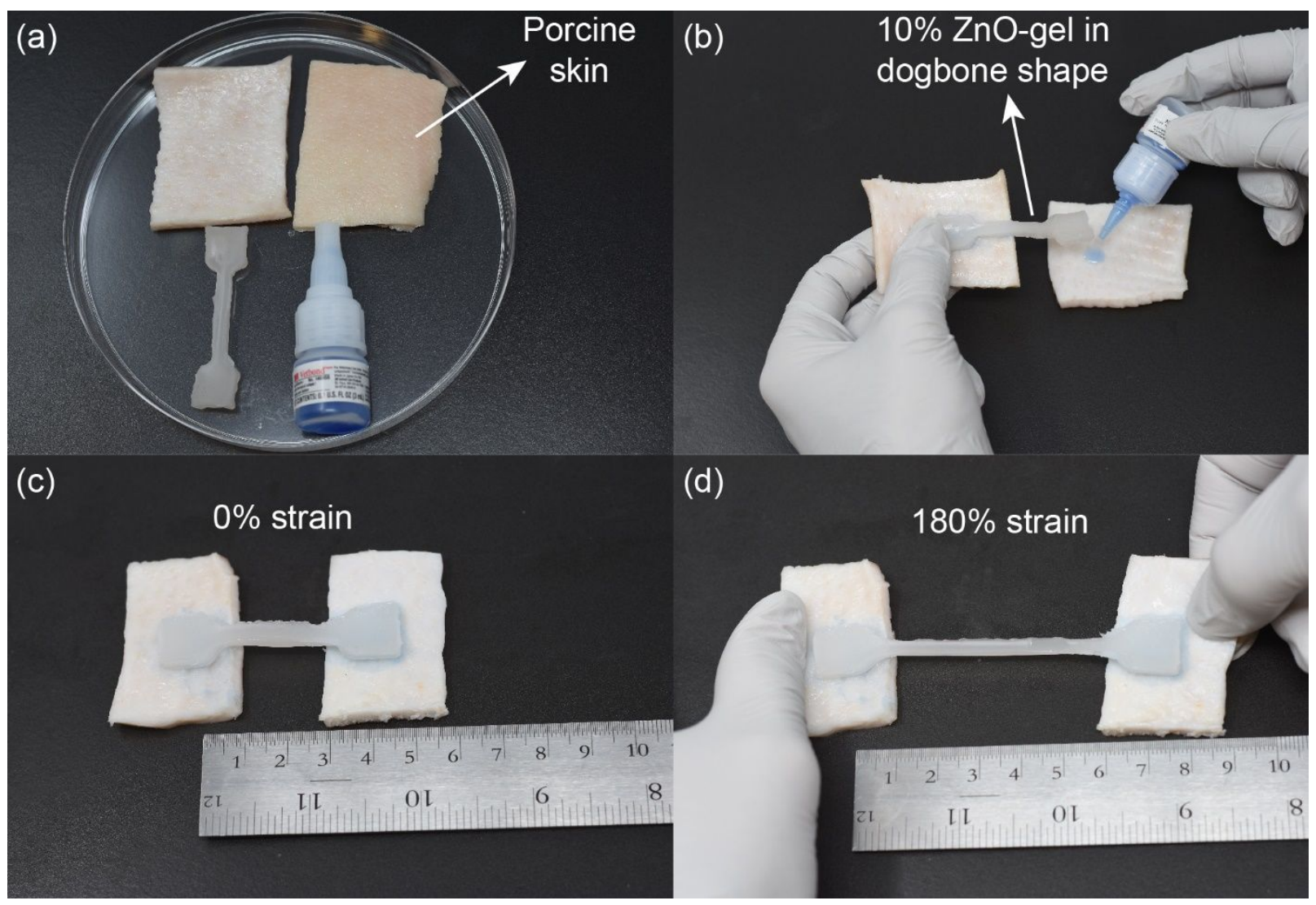

Figure S4. Demonstration of attaching 10\% ZnO-gel onto thick porcine skin (a-b) by using a commercial tissue adhesive. Photograph of the bonded hydrogel (c) before stretching and (d) after stretching to $180 \%$ strain. 


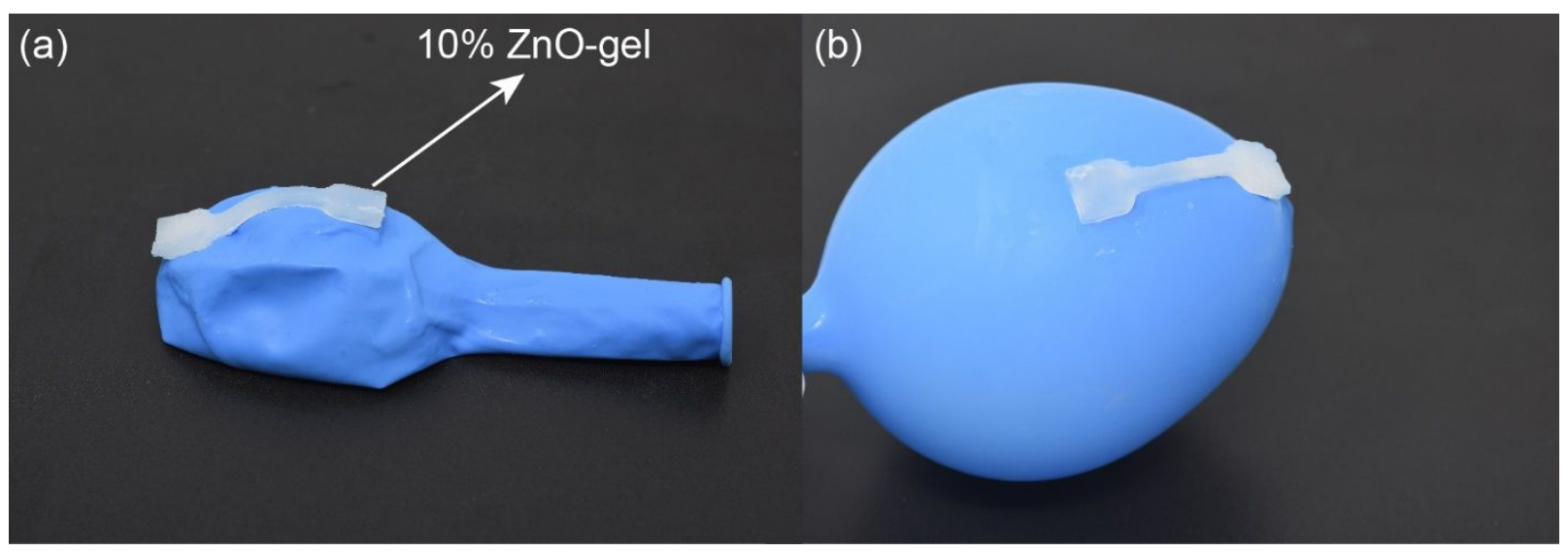

Figure S5. Demonstration of 10\% ZnO-gel attachment on a balloon via surgical adhesive. Photograph of (a) hydrogel attachment onto balloon surface before inflating and (b) after inflating and stretching the hydrogel. 